\title{
A Korean patient with Guillain-Barré syndrome follow- ing acute hepatitis $E$ whose cholestasis resolved with steroid therapy
}

\author{
Sung Bok Ji', Sang Soo Lee', Hee cheul Jung ${ }^{1}$, Hong Jun Kim¹, Hyun Jin Kim', Tae Hyo Kim', Woon Tae Jung', \\ Ok Jae Lee', Dae Hyun Song ${ }^{2}$ \\ 1Department of Internal Medicine, Gyeongsang National University Hospital, Gyeongsang National University School of Medicine, Jinju; \\ ${ }^{2}$ Department of Pathology, Gyeongsang National University Hospital, Gyeongsang National University School of Medicine, Jinju, Korea
}

\begin{abstract}
Autochthonous hepatitis E virus (HEV) is an emerging pathogen in developed countries, and several cases of acute HEV infection have been reported in South Korea. However, there have been no reports on HEV-associated Guillain-Barré syndrome (GBS) in Korea. We recently experienced the case of a 58-year-old Korean male with acute HEV infection after ingesting raw deer meat. Persistent cholestasis was resolved by the administration of prednisolone. At 2.5 months after the clinical presentation of HEV infection, the patient developed weakness of the lower limbs, and was diagnosed with GBS associated with acute hepatitis E. To our knowledge, this is the second report on supportive steroid therapy for persistent cholestasis due to hepatitis $\mathrm{E}$, and the first report of GBS in a Korean patient with acute HEV infection.
\end{abstract}

(Clin Mol Hepatol 2016;22:396-399)

Keywords: Hepatitis E; Jaundice; Steroid treatment; Guillain-Barré syndrome

\section{INTRODUCTION}

Hepatitis E virus (HEV) infection is an emerging problem in developed countries, but HEV is a rare cause of acute viral hepatitis in South Korea.' Several cases of acute hepatitis E infection have been reported in Korea. ${ }^{2-8}$ Most patients of acute hepatitis $\mathrm{E}$ infection are self-limiting and require no treatment. Moreover, a small number of patients with acute HEV genotype 1 or 3 infection have been treated with antiviral therapy. ${ }^{9-11}$ However, reports on the clinical significance of use of steroid in patients with cholestatic hepatitis $E$ are very limited.

Guillain-Barré syndrome (GBS) is triggered by a preceding infec- tion including acute hepatitis A, B, and C. Occasionally, it has been triggered by HEV infection. ${ }^{12-14}$

We reported a case of persistent cholestasis caused by an autochthonous HEV infection that was resolved with steroid treatment. After 2.5 months, the patients developed weakness of the lower limbs, and were diagnosed with GBS associated with acute hepatitis $\mathrm{E}$.

\section{CASE REPORT}

On 20 March 2014, a 58-years-old Korean male was referred to

\section{Abbreviations:}

ALT, alanine aminotransferase; AST, aspartate aminotransferase; CSF, cerebrospinal fluid; GBS, Guillain-Barré syndrome; HAV, hepatitis A virus; HEV, hepatitis E virus; Ig, immunoglobulin

\author{
Corresponding author: Sang Soo Lee \\ Department of Internal Medicine, Gyeongsang National University \\ Hospital, Gyeongsang National University School of Medicine, 79 \\ Gangnam-ro, Jinju 52727, Korea \\ Tel: +82-55-750-8932, Fax: +82-55-750-9496 \\ E-mail:3939lee@naver.com
}



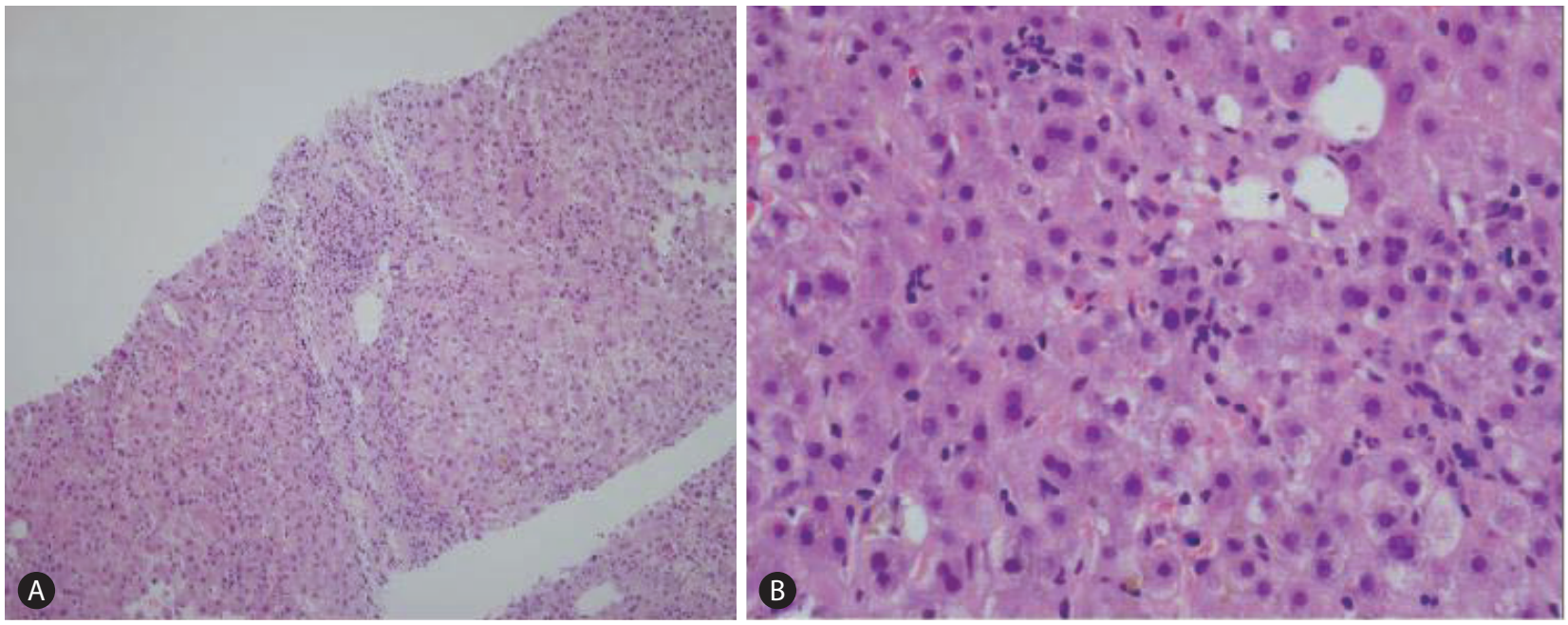

Figure 1. Liver biopsy of acute hepatitis E. (A) Inflammatory cells had accumulated in the periportal area. There was no fatty change. Swelling and focal apoptosis of hepatocytes were present. Periportal fibrosis with bridging was observed $(H \& E, \times 100)$. (B) Lymphocytes were the dominant inflammatory cells in the portal area $(H \& E, \times 400)$.

our hospital with severe hepatitis of unknown cause. On 10 March 2014, the patient had presented with anorexia, pruritus, and jaundice. He was a heavy alcohol drinker, with a consumption rate of $120 \mathrm{~g} /$ day of alcohol for 30 years. He had not travelled outside South Korea. There was no history of blood transfusions, risky sexual behavior or drug addiction. Three months before hospitalization, he ingested raw deer meat with the intention of improving his stamina. He had ingested about $200 \mathrm{~g}$ of raw meat obtained from a wild deer captured on Jiri-mountain in the Gyeongnam province, South Korea.

On physical examination, he had jaundice, right upper quadrant tenderness and an enlarged liver, but showed no feature of hepatic encephalopathy. Initial laboratory data showed white blood cell count of $6.36 \times 10^{3} / \mathrm{mm}^{3}$ (polymorphonuclear neutrophils, $60.1 \%$; lymphocytes, $24.1 \%$; and eosinophils, $2.2 \%$ ), serum total bilirubin level of $23.59 \mathrm{mg} / \mathrm{dL}$, serum aspartate aminotransferase (AST) level of $292 \mathrm{IU} / \mathrm{L}$, and serum alanine aminotransferase (ALT) level of $525 \mathrm{IU} / \mathrm{L}$. Prothrombin time, electrolytes and renal function tests were normal. Serologic study was negative for immunoglobulin (Ig) M anti-hepatitis A virus (HAV) antibody and positive for IgG anti-HAV antibody. Hepatitis B virus surface antigen and antibody to hepatitis $C$ were absent, and HCV RNA was negative. Abdominal computed tomography showed findings compatible with chronic liver disease with splenomegaly.

On the ninth day of admission, laboratory data showed a peak total bilirubin level of $>35.0 \mathrm{mg} / \mathrm{dL}$ and liver biopsy and blood test for IgM and IgG anti-HEV (Dia. Pro, Milan, Italy for IgG anti-HEV ELISA and DSI, Milan, Italy for IgM anti-HEV ELISA) were per-

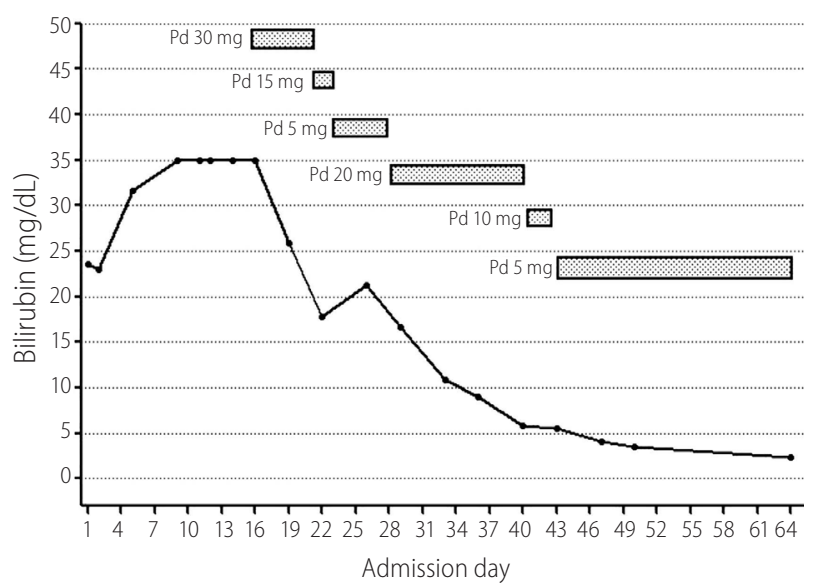

Figure 2. Total bilirubin levels before and after steroid treatment. The steroid was started at day 16 , after which the total bilirubin level decreased.

formed. On liver biopsy (Fig. 1), the lymphocyte-dominant inflammatory cells were accumulated in the periportal area. There was no fatty change. Swelling and focal apoptosis of hepatocytes were present. Periportal fibrosis with bridge necrosis was observed. Sixteen days after admission, we started $30 \mathrm{mg} /$ day of prednisolone despite the normal prothrombin time because of a steady increase in total bilirubin levels and pruritus. On the fourteen day of admission, commercially available immunoassay for IgM anti-HEV and IgG anti-HEV were both positive. The optical density value of IgM anti-HEV was 0.849 (cut-off value 0.294) and IgG anti-HEV was 3.356 (cut-off value: 0.367).

At the time of the diagnosis of acute HEV, we decided to taper 
steroid therapy, but the total bilirubin level was elevated $(17.8 \mathrm{mg} /$ $\mathrm{dL}$ to $21.3 \mathrm{mg} / \mathrm{dL}$ ) after steroid tapering (30 mg/day to $5 \mathrm{mg} /$ day over 10 days) (Fig. 2). Thereafter, prednisolone was given at a dosage of $20 \mathrm{mg} / \mathrm{day}$, and the dose of steroid was tapered and discontinued after 5 weeks. Two months after admission, laboratory data showed a total bilirubin level of $2.30 \mathrm{mg} / \mathrm{dl}$, AST level of $24 \mathrm{IU} / \mathrm{L}$, and ALT level of $26 \mathrm{IU} / \mathrm{L}$ (Fig. 2).

On the seventy ninth day of admission, the patient developed weakness of the lower limbs. The muscle weakness progressed rapidly and in 2 days he was bedridden, unable to move against gravity. There was no other infection associated with GBS. Cerebrospinal fluid (CSF) analysis showed a normal protein concentration $(44.6 \mathrm{mg} / \mathrm{dl})$ without cellular response. Nerve-conduction studies showed findings compatible with GBS. Intravenous immunoglobulin $30 \mathrm{~g} /$ day was administered for 5 days, and resulted in significantly improved neurologic symptom. During the follow-up period, IgM anti-HEV persisted as positive until 6 months after admission (value 1.883), but IgM anti-HEV converted negative on 12 months after admission (value 0.049 ).

\section{DISCUSSION}

At first, we considered his severe jaundice may be a manifestation of alcoholic hepatitis on underlying alcoholic cirrhosis. The diagnosis of acute hepatitis E infection was delayed because we performed blood tests for IgM and IgG anti-HEV due to persistent jaundice on the ninth day of administration. Steroid therapy with prednisolone was started on the sixteenth day, because of pruritus and the possibility of acute-on-chronic liver failure with progressive liver injury. Immunosuppressive treatment for acute viral hepatitis seems to be counterproductive, hence prednisolone was tapered to $5 \mathrm{mg} / \mathrm{day}$. Since the total bilirubin level was elevated after tapering, we decided to continue prednisolone treatment at $20 \mathrm{mg} / \mathrm{day}$. Two months after admission, the patients liver function was almost normalized. An AST/ALT ratio $<1$ and periportal lymphocyte infiltration in the absence of fatty liver, neutrophil infiltration, and Mallory bodies suggest that severe jaundice may be caused by viral-induced cholestasis, not alcoholic hepatitis in our case.

To our knowledge, this is the second report on supportive steroid therapy for severe acute HEV infection. Sebode et al. ${ }^{15}$ reported an acute HEV infection case with improved acute liver injury under steroid treatment. They showed that steroid treatment might be an option to prevent the progression of liver failure in patients with HEV induced acute liver injury.

In a recent study, ${ }^{14} 5 \%$ of patients with GBS had an associated acute HEV infection in the Netherlands. In Korea, reported HEV data on seroprevalence of IgG HEV ranges from $17 \%$ to $27 \%$. However, there has been no report on acute HEV complicated by GBS in Korea, and this is the first report of GBS in a hepatitis E patient in Korea. Therefore, further studies are required to determine the frequency of antecedent HEV infection in patients with GBS in other geographical areas including Korea. Extrahepatic symptoms may develop even after the clearance of HEV virus and not only during active HEV infection. ${ }^{16}$ In our case, the neurologic symptom developed at 2.5 months after acute HEV infection.

HEV RNA becomes undetectable in blood about 3 weeks after symptom onset with reported sensitivity of HEV IgM tests between $72 \%$ and $98 \%$ and specificities between $78.2 \%$ and 95.6\%. ${ }^{17}$ During the follow-up period, IgM anti-HEV persisted as positive thrice until 1 October 2014, but IgM anti-HEV converted to negative on 12 months after admission. Thus, this test can be used to confirm the diagnosis of HEV infection. There was a major limitation for the interpretation in our case. Recovery of severe cholestasis might just reflect the natural course of HEV infection without steroid treatment. However, figure 2 showed that serum bilirubin level was promptly decreased following treatment of steroid. In addition, it is possible that steroid therapy had some influence on the development of GBS. ${ }^{18}$

In conclusion, this case suggested that steroid therapy might be a beneficial option to relieve pruritus related to persistent cholestasis complicated with acute hepatitis E infection. In addition, further investigations is needed to assess the prevalence of HEV infection in patients with GBS.

\section{Conflicts of Interest}

The authors have no conflicts to disclose.

\section{REFERENCES}

1. Jeong SH. Current status of hepatitis e virus infection in Korea. Gut Liver 2011;5:427-431.

2. Kim NJ, Lee JS, Kim KA, Lee HR, Oh JW, Cho YD, et al. [A case of acute hepatitis E]. Taehan Kan Hakhoe Chi 2002;8:312-316.

3. Kim SS, Yeo CD, Kim JD, Kim HS, Jang JW, Kim CW, et al. [A case of acute hepatitis $\mathrm{E}$ with the travel history to an endemic area]. Korean J Gastroenterol 2004;44:342-345.

4. Kong SJ, Min SK, Kim IK, Koo H, Park, II, Han JP, et al. [Two cases of acute hepatitis $\mathrm{E}$ in patients with hyperthyroidism]. Korean J Gastroenterol 2006;47:65-71. 
5. Lim JW, Park CS, Ahn JM, Yu MH, Kim TS, Lim YS, et al. [Nine cases of sporadic acute hepatitis $\mathrm{E}$ in Korea]. Korean J Hepatol 2006;12:230-236

6. Kim DH, Park H, Moon SW, Jeong JH, Yang HS, Kim DH, et al. [Three sporadic cases of acute hepatitis E]. Korean J Gastroenterol 2007:50:121-125.

7. Choi JY, Lee JM, Jo YW, Min HJ, Kim HJ, Jung WT, et al. Genotype-4 hepatitis $\mathrm{E}$ in a human after ingesting roe deer meat in South Korea. Clin Mol Hepatol 2013;19:309-314.

8. Kim YM, Jeong SH, Kim JY, Song JC, Lee JH, Kim JW, et al. The first case of genotype 4 hepatitis $\mathrm{E}$ related to wild boar in South Korea. J Clin Virol 2011;50:253-256.

9. Gerolami R, Borentain P, Raissouni F, Motte A, Solas C, Colson P. Treatment of severe acute hepatitis $E$ by ribavirin. J Clin Virol 2011;52:60-62.

10. Goyal R, Kumar A, Panda SK, Paul SB, Acharya SK. Ribavirin therapy for hepatitis $E$ virus-induced acute on chronic liver failure: a preliminary report. Antivir Ther 2012;17:1091-1096.

11. Péron JM, Dalton H, Izopet J, Kamar N. Acute autochthonous hepatitis $E$ in western patients with underlying chronic liver disease: a role for ribavirin? J Hepatol 2011;54:1323-1324.
12. Sood A, Midha V, Sood N. Guillain-Barré syndrome with acute hepatitis E. Am J Gastroenterol 2000;95:3667-3668.

13. Loly JP, Rikir E, Seivert M, Legros E, Defrance P, Belaiche J, et al. Guillain-Barré syndrome following hepatitis E. World J Gastroenterol 2009;15:1645-1647.

14. van den Berg B, van der Eijk AA, Pas SD, Hunter JG, Madden RG, Tio-Gillen AP, et al. Guillain-Barré syndrome associated with preceding hepatitis E virus infection. Neurology 2014;82:491-497.

15. Sebode M, Pischke S, Lütgehetmann M, Polywka S, Quaas A, Lohse $A W$, et al. New foe treated with old guns - supportive role of steroids in the treatment of acute severe hepatitis E. BMC Gastroenterol 2014;14:191.

16. Aggarwal R. Clinical presentation of hepatitis E. Virus Res 2011;161:15-22.

17. Drobeniuc J, Meng J, Reuter G, Greene-Montfort T, Khudyakova N, Dimitrova Z, et al. Serologic assays specific to immunoglobulin $\mathrm{M}$ antibodies against hepatitis $\mathrm{E}$ virus: pangenotypic evaluation of performances. Clin Infect Dis 2010;51:e24-27.

18. Steiner I, Wirguin I, Abramsky 0. Appearance of Guillain-Barre syndrome in patients during corticosteroid treatment. J Neurol 1986;233:221-223. 